\title{
Multiplicity fluctuations in hadronic final states from the decay of the $\mathrm{Z}^{0}$
}

\section{DELPHI Collaboration}

P. Abreu "a, W. Adam ', T. Adye " , E. Agasi ' , G.D. Alekseev ${ }^{\text {e }}$, A. Algeri ${ }^{\mathrm{f}}$, P. Allen ${ }^{g}$, S. Almehed ${ }^{\text {h }}$, S.J. Alvsvaag ${ }^{i}$, U. Amaldi ${ }^{j}$, E.G. Anassontzis ${ }^{k}$, A. Andreazza ', P. Antilogus ', W.-D. Apel " , R.J. Apsimon c, B. Åman ", J-E. Augustin ${ }^{\text {p }, ~ A . ~ A u g u s t i n u s ~}{ }^{d}$, P. Baillon ${ }^{\text {, }}$, P. Bambade ${ }^{\text {P }}$, F. Barao ", R. Barate ", G. Barbiellini ', D.Y. Bardin ', G. Barker ", A. Baroncelli ", O. Barring ", J.A. Barrio ", W. Bartl ", M.J. Bates c, M. Battaglia ${ }^{\text {', }}$

M. Baubillier ${ }^{\text {v }}$, K-H. Becks w, C.J. Beeston ", M. Begalli ${ }^{x}$, P. Beilliere ${ }^{y}$, Yu. Belokopytov ${ }^{\text {z }}$, P. Beltran ${ }^{\text {ala }}$, D. Benedic ab, A.C. Benvenuti ${ }^{\text {ac }}$, M. Berggren ${ }^{\text {p }}$, D. Bertrand ${ }^{\text {ad }}$, F. Bianchi ae, M.S. Bilenky ${ }^{\mathrm{e}}$, P. Billoir ${ }^{ }$, J. Bjarne ${ }^{\mathrm{h}}$, D. Bloch ${ }^{\mathrm{ab}}$,

S. Blyth ", V. Bocci af , P.N. Bogolubov e, T. Bolognese ag, M. Bonesini ', W. Bonivento ${ }^{\text {, P.S.L. Booth }}{ }^{\text {ah }}$, P. Borgeaud ${ }^{\text {ag }}$, G. Borisov ${ }^{2}$, H. Borner ${ }^{j}$,

C. Bosio ', B. Bostjancic ${ }^{\text {, }}$, S. Bosworth s, O. Botner ${ }^{\text {ai, B. Bouquet }}{ }^{\mathrm{p}}$, C. Bourdarios ${ }^{p}$, T.J.V. Bowcock ${ }^{\text {ah }}$, M. Bozzo aj, S. Braibant ad, P. Branchini ${ }^{\text {, }}$, K.D. Brand ${ }^{\text {ak }}$, R.A. Brenner ${ }^{\text {i, H. Briand }}{ }^{\text {v }}$, C. Bricman ad, R.C.A. Brown ${ }^{\text {j, }}$

N. Brummer ${ }^{\mathrm{d}}$, J-M. Brunet ${ }^{\mathrm{y}}$, L. Bugge ${ }^{\text {al }}$, T. Buran ${ }^{\text {al }}$, H. Burmeister ${ }^{\text {, }}$, J.A.M.A. Buytaert ${ }^{\mathrm{j}}$, M. Caccia ${ }^{\mathrm{j}}$, M. Calvi ${ }^{\text {, }}$, A.J. Camacho Rozas ${ }^{\text {am }}$,

T. Camporesi i, V. Canale ${ }^{\text {af }}$, F. Cao ${ }^{\text {ad }}$, F. Carena ${ }^{j}$, L. Carroll ${ }^{\text {ah }}$, C. Caso ${ }^{\text {aj }}$, M.V. Castillo Gimenez ${ }^{\mathrm{g}}$, A. Cattai ${ }^{\mathrm{j}}$, F.R. Cavallo ${ }^{\text {ac }}$, L. Cerrito ${ }^{\text {af }}$, V. Chabaud ${ }^{\mathrm{i}}$,

A. Chan ${ }^{\text {an }}$, Ph. Charpentier ${ }^{j}$, L. Chaussard ${ }^{\mathrm{p}}$, J. Chauveau ${ }^{\text {, }}$ P. Checchia ${ }^{\mathrm{ak}}$, G.A. Chelkov ${ }^{\text {e }, ~ L . ~ C h e v a l i e r ~ a g, ~ P . ~ C h l i a p n i k o v ~ ", ~ V . ~ C h o r o w i c z ~ ", ~ J . T . M . ~ C h r i n ~ g, ~}$ M.P. Clara ${ }^{\text {ae }}$, P. Collins s", J.L. Contreras ", R. Contri ${ }^{\text {aj }}$, E. Cortina ${ }^{\text {g }}$, G. Cosme ${ }^{\text {p }}$, F. Couchot ${ }^{\mathrm{p}}$, H.B. Crawley an, D. Crennell ${ }^{c}$, G. Crosetti ${ }^{\mathrm{aj}}$, M. Crozon ${ }^{\mathrm{y}}$, J. Cuevas Maestro am, S. Czellar ${ }^{\text {}}$, E. Dahl-Jensen ${ }^{\text {ao }}$, B. Dalmagne ${ }^{\mathrm{p}}$, M. Dam ${ }^{\text {al }}$, G. Damgaard "o, G. Darbo aj, E. Daubie ad, A. Daum ", P.D. Dauncey ", M. Davenport ' , P. David v, W. Da Silva v, C. Defoix `, D. Delikaris ', S. Delorme ${ }^{j}$, P. Delpierre ${ }^{y}$, N. Demaria ae ${ }^{\text {ae }}$ A. De Angelis ${ }^{r}$, M. De Beer ag, H. De Boeck ${ }^{\text {ad }}$, W. De Boer ${ }^{\text {n, C. De Clercq }}{ }^{\text {ad }}$, M.D.M. De Fez Laso ${ }^{\text {g }}$, N. De Groot ${ }^{d}$, C. De La Vaissiere ${ }^{\vee}$, B. De Lotto ${ }^{r}$, A. De Min ', H. Dijkstra ${ }^{j}$, L. Di Ciaccio af , F. Djama ab, J. Dolbeau ${ }^{y}$, M. Donszelmann ${ }^{j}$, K. Doroba ap, M. Dracos ${ }^{\text {i }}$, J. Drees ${ }^{\text {w }}$, M. Dris ${ }^{\mathrm{aq}}$, Y. Dufour ${ }^{y}$, L-O. Eek ${ }^{\text {ai }}$, P.A.-M. Eerola ${ }^{j}$, R. Ehret ${ }^{n}$, T. Ekelof ai, G. Ekspong ${ }^{\circ}$, A. Elliot Peisert ${ }^{a k}$, J-P. Engel ${ }^{a b}$, D. Fassouliotis aq, T.A. Fearnley ${ }^{i}$, M. Feindt ${ }^{i}$, M. Fernandez Alonso ${ }^{\text {am }}$, 
A. Ferrer ${ }^{\mathrm{q}}$, T.A. Filippas ${ }^{\mathrm{aq}}$, A. Firestone ${ }^{\text {an }}$, H. Foeth ${ }^{j}$, E. Fokitis ${ }^{\text {aq }}$, F. Fontanelli aj, K.A.J. Forbes ${ }^{\mathrm{ah}}$, J-L. Fousset ${ }^{\text {ar }}$, S. Francon ${ }^{\mathrm{m}}$, B. Franek ${ }^{\mathrm{c}}$, P. Frenkiel ${ }^{y}$, D.C. Fries ${ }^{n}$, A.G. Frodesen ${ }^{i}$, R. Fruhwirth ${ }^{b}$, F. Fulda-Quenzer ${ }^{p}$, K. Furnival ah, H. Furstenau ${ }^{\text {n, J. Fuster }}{ }^{j}$, G. Galeazzi ${ }^{\text {ak }}$, D. Gamba ae, C. Garcia ${ }^{g}$, J. Garcia am, C. Gaspar j, U. Gasparini ak, Ph. Gavillet j, E.N. Gazis ${ }^{a q}$, J-P. Gerber ${ }^{\text {ab }}$, P. Giacomelli ${ }^{j}$, R. Gokieli ${ }^{\text {ap }}$, B. Golob as, V.M. Golovatyuk e, J.J. Gomez Y Cadenas ', A. Goobar ', G. Gopal c, M. Gorski ap, V. Gracco aj, A. Grant ' , F. Grard ad, E. Graziani ',

G. Grosdidier ${ }^{\text {p }}$, E. Gross ${ }^{\text {i }, ~ P . ~ G r o s s e-W i e s m a n n ~}{ }^{\text {j }}$, B. Grossetete ${ }^{\text {v }}$, J. Guy ${ }^{\text {c }}$, U. Haedinger " , F. Hahn ${ }^{\text {w }}$, M. Hahn ${ }^{\text {", S. Haider }}{ }^{\text {d, Z. Hajduk }}{ }^{\text {at }}$, A. Hakansson ${ }^{\text {h, }}$, A. Hallgren ai, K. Hamacher ${ }^{\text {w }}$, G. Hamel De Monchenault ag, W. Hao d,

F.J. Harris s, T. Henkes ${ }^{\text {j }}$, J.J. Hernandez ${ }^{g}$, P. Herquet ad , H. Herr ${ }^{\text {j, }}$, T.L. Hessing ah, I. Hietanen ${ }^{\text {f }}$, C.O. Higgins ${ }^{\text {ah }}$, E. Higon ${ }^{g}$, H.J. Hilke ${ }^{j}$,

S.D. Hodgson s, T. Hofmokl ap, R. Holmes an, S-O. Holmgren ", D. Holthuizen ${ }^{\text {d }}$, P.F. Honore ${ }^{y}$, J.E. Hooper ${ }^{\text {to }}$, M. Houlden ${ }^{\text {ah }}$, J. Hrubec ${ }^{\text {b }}$, C. Huet ad, P.O. Hulth ${ }^{\circ}$, K. Hultqvist ${ }^{\circ}$, P. Ioannou ${ }^{k}$, D. Isenhower ${ }^{j}$, P-S. Iversen ${ }^{i}$,

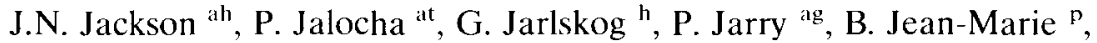
E.K. Johansson ${ }^{\circ}$, D. Johnson ${ }^{\text {ah }}$, M. Jonker ${ }^{j}$, L. Jonsson ${ }^{\text {h }}$, P. Juillot ${ }^{\mathrm{ab}}$, G. Kalkanis " , G. Kalmus ' , F. Kapusta v, M. Karlsson ', E. Karvelas "ai, S. Katsanevas ${ }^{k}$, E.C. Katsoufis ${ }^{a q}$, R. Keranen ${ }^{f}$, J. Kesteman ad, B.A. Khomenko ${ }^{e}$, N.N. Khovanski ${ }^{\text {e }}$, B. King ${ }^{\text {ah }}$, N.J. Kjaer ${ }^{j}$, H. Klein ${ }^{j}$, W. Klempt ${ }^{\text {j }}$, A. Klovning ${ }^{\text {i }}$, P. Kluit ${ }^{\text {d }}$, A. Koch-Mehrin ${ }^{\text {w }}$, J.H. Koehne ${ }^{\text {", }}$, B. Koene ${ }^{\mathrm{d}}$, P. Kokkinias ${ }^{\text {aa }}$, M. Kopf ", K. Korcyl at, A.V. Korytov ${ }^{\mathrm{e}}$, V. Kostioukhine ${ }^{\text {, }}$ C. Kourkoumelis ${ }^{\text {k }}$, O. Kouznetsov e, P.H. Kramer ${ }^{\text {w, }}$ J. Krolikowski ap, I. Kronkvist ${ }^{\text {h }}$, U. Kruener-Marquis ${ }^{\text {w }}$, W. Krupinski at, K. Kulka ${ }^{\text {ai }}$, K. Kurvinen ${ }^{\text {f }}$, C. Lacasta ${ }^{\text {g }}$, C. Lambropoulos aa , J.W. Lamsa an, L. Lanceri r, V. Lapin " , J-P. Laugier ag, R. Lauhakangas ', G. Leder ' F. Ledroit ${ }^{\mathrm{q}}$, R. Leitner ${ }^{\mathrm{j}}$, Y. Lemoigne ${ }^{\mathrm{ag}}$, J. Lemonne ${ }^{\mathrm{ad}}$, G. Lenzen ${ }^{\text {w }}$,

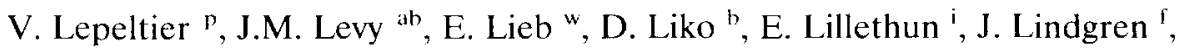
R. Lindner ${ }^{\text {w }}$, A. Lipniacka ${ }^{\text {ap }}$, I. Lippi ${ }^{\text {ak }}$, B. Loerstad ${ }^{\text {h }}$, M. Lokajicek ${ }^{\text {c }}$,

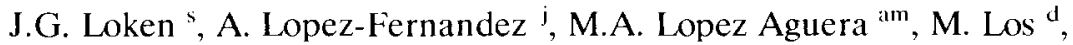
D. Loukas aa, J.J. Lozano ${ }^{\text {g }}$, P. Lutz ${ }^{\text {y }}$, L. Lyons s, G. Machlum ${ }^{\text {al }}$, J. Maillard ${ }^{\text {, }}$ A. Maltezos ${ }^{\text {aa }}$, F. Mandl ${ }^{\text {b }}$, J. Marco ${ }^{\text {am }}$, M. Margoni ${ }^{\text {ik }}$, J-C. Marin ${ }^{\text {j, }}$,

A. Markou aa , T. Maron ${ }^{\text {w }}$, S. Marti ${ }^{\text {g }}$, L. Mathis ${ }^{\text {an }}$, F. Matorras ${ }^{\text {am }}$, C. Matteuzzi ${ }^{1}$, G. Matthiae af ${ }^{\text {af }}$ M. Mazzucato ${ }^{a k}$, M. Mc Cubbin ah, R. Mc Kay an, R. Mc Nulty ${ }^{\text {ah }}$, G. Meola aj, C. Meroni ' ${ }^{\text {', W.T. Meyer }}{ }^{\text {an, }}$, M. Michelotto ${ }^{a k}$, I. Mikulec ${ }^{\text {b }}$, L. Mirabito ${ }^{m}$, W.A. Mitaroff ${ }^{\text {b }}$, G.V. Mitselmakher ${ }^{\text {, }}$ U. Mjoernmark ', T. Moa ${ }^{\text {, }}$, R. Moeller ao, K. Moenig ${ }^{\text {, }}$ M.R. Monge aj, P. Morettini aj, H. Mueller ${ }^{\text {n }}$, W.J. Murray ${ }^{\text {c, B. Muryn }}{ }^{\text {at }}$,

G. Myatt ${ }^{\text {s }}$, F. Naraghi ${ }^{\vee}$, F.L. Navarria ${ }^{a c}$, P. Negri ${ }^{1}$, B.S. Nielsen ${ }^{\text {ao }}$, B. Nijjhar ${ }^{\text {ah }}$, V. Nikolaenko ${ }^{\text {z }}$, P.E.S. Nilsen i, P. Niss ", V. Obraztsov ${ }^{\text {", }}$, A.G. Olshevski ${ }^{\text {c }}$, R. Orava ${ }^{f}$, A. Ostankov ${ }^{z}$, K. Osterberg ${ }^{f}$, A. Ouraou ${ }^{a g}$, 
M. Paganoni ', R. Pain ${ }^{\text {v }}$, H. Palka ${ }^{\text {d }}$, Th.D. Papadopoulou aq , L. Pape ${ }^{\text {i }}$, A. Passeri ${ }^{t}$, M. Pegoraro ${ }^{\text {ak }}$, J. Pennanen ${ }^{f}$, V. Perevozchikov ${ }^{z}$, M. Pernicka ${ }^{b}$, A. Perrotta ${ }^{\text {ac }}$, C. Petridou ${ }^{r}$, A. Petrolini ${ }^{\text {aj }}$, T.E. Pettersen ${ }^{\text {ak }}$, F. Pierre ${ }^{a g}$, M. Pimenta ", O. Pingot ${ }^{\text {ad }}$, M.E. Pol ${ }^{j}$, G. Polok ${ }^{\text {at }}$, P. Poropat ${ }^{\mathrm{r}}$, P. Privitera ", A. Pullia ', D. Radojicic ', S. Ragazzi ', H. Rahmani a4, P.N. Ratoff ${ }^{\text {itu, }}$ A.L. Read al, N.G. Redaelli ', M. Regler ", D. Reid 'ah, P.B. Renton ", L.K. Resvanis ${ }^{k}$, F. Richard ${ }^{\text {p }}$, M. Richardson ${ }^{\text {ah }}$, J. Ridky ${ }^{\text {e }}$, G. Rinaudo ae, I. Roditi ${ }^{\text {av }}$, A. Romero ${ }^{\text {ae }}$, I. Roncagliolo ${ }^{a j}$, P. Ronchese ${ }^{a k}$, C. Ronnquist ${ }^{f}$, E.I. Rosenberg an, S. Rossi ${ }^{j}$, U. Rossi ${ }^{\text {ac }}$, E. Rosso ${ }^{j}$, P. Roudeau ${ }^{\text {p }}$, T. Rovelli "ac, W. Ruckstuhl `, V. Ruhlmann-Kleider "ag, A. Ruiz " ${ }^{\text {am }}$, K. Rybicki ${ }^{\text {at }}$, H. Saarikko ${ }^{\text {f }}$, Y. Sacquin ${ }^{\text {ag }}$, G. Sajot ${ }^{\text {q }}$, J. Salt ${ }^{\mathrm{g}}$, J. Sanchez ", M. Sannino aj, S. Schael ", H. Schneider ", M.A.E. Schyns ${ }^{\text {w }}$, G. Sciolla ${ }^{\text {ae }}$, F. Scuri ${ }^{\text {r }}$ A.M. Segar ", R. Sekulin ${ }^{\text {c }}$, M. Sessa ${ }^{\text {, }}$, G. Sette ${ }^{\text {ij }}$, R. Seufert ", R.C. Shellard ${ }^{x}$, I. Siccama ${ }^{d}$, P. Siegrist ${ }^{\text {ag }}$, S. Simonetti ${ }^{\text {aj, F. Simonetto }}{ }^{\mathrm{ak}}$,

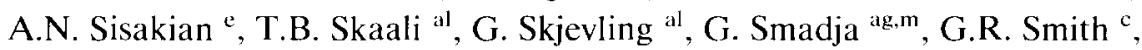
R. Sosnowski ${ }^{j}$, T.S. Spassoff ${ }^{4}$, E. Spiriti ', S. Squarcia ${ }^{\text {aj }}$, H. Staeck ${ }^{\text {w }}$, C. Stanescu ${ }^{\text {t, S. Stapnes }}{ }^{\text {al }}$, G. Stavropoulos a ${ }^{\text {aa }}$, F. Stichelbaut ad, A. Stocchi ${ }^{\text {, }}$, J. Strauss ' ${ }^{\text {, J. Straver }}{ }^{\text {, }}$, R. Strub ${ }^{\text {ab }}$, M. Szczekowski ${ }^{\text {, }}$, M. Szeptycka ap, P. Szymanski ap, T. Tabarelli ${ }^{1}$, S. Tavernier ad , O. Tchikilev ${ }^{z}$, G.E. Theodosiou aa, A. Tilquin ${ }^{\text {ar }}$, J. Timmermans ${ }^{\text {d }, ~ V . G . ~ T i m o f e e v ~}{ }^{\text {e }}$, L.G. Tkatchev ${ }^{\mathrm{e}}$, T. Todorov ${ }^{\mathrm{ab}}$, D.Z. Toet ${ }^{d}$, O. Toker ${ }^{f}$, E. Torassa ${ }^{\text {aj }}$, L. Tortora ', D. Treille ${ }^{j}$, U. Trevisan $^{\text {aj, }}$, W. Trischuk ${ }^{j}$, G. Tristram ${ }^{y}$, C. Troncon ', A. Tsirou ', E.N. Tsyganov ${ }^{c}$, M. Turala a', M-L. Turluer ag, T. Tuuva ', I.A. Tyapkin ${ }^{\text {v }, ~ M . ~ T y n d e l ~ ", ~}$ S. Tzamarias ${ }^{\text {j }}$, S. Ueberschaer ${ }^{\text {w }}$, O. Ullaland ${ }^{j}$, V. Uvarov ${ }^{\text {z }}$, G. Valenti ${ }^{\text {ac }}$, E. Vallazza " ${ }^{\text {" }}$ J.A. Valls Ferrer ${ }^{\mathrm{a}}$, C. Vander Velde ${ }^{\text {ad }}$, G.W. Van Apeldoorn ${ }^{\mathrm{d}}$, P. Van Dam ${ }^{d}$, M. Van Der Heijden ${ }^{d}$, W.K. Van Doninck ${ }^{\text {ad }}$, P. Vaz ${ }^{j}$, G. Vegni ', L. Ventura ${ }^{\text {ak }}$, W. Venus ${ }^{c}$, F. Verbeure ${ }^{\text {ad }}$, L.S. Vertogradov ${ }^{\mathrm{e}}$, D. Vilanova ${ }^{\text {ag }}$, P. Vincent ${ }^{\mathrm{m}}$, L. Vitale ${ }^{\mathrm{f}}$, E. Vlasov ${ }^{\mathrm{z}}$, A.S. Vodopyanov ${ }^{\mathrm{c}}$, M. Vollmer ${ }^{\mathrm{w}}$, G. Voulgaris ${ }^{k}$, M. Voutilainen ${ }^{\mathrm{f}}$, V. Vrba ${ }^{\mathrm{t}}$, H. Wahlen ${ }^{\mathrm{w}}$, C. Walck ${ }^{\circ}$,

F. Waldner ${ }^{r}$, M. Wayne ${ }^{\text {an }}$, A. Wehr ${ }^{w}$, M. Weierstall ${ }^{w}$, P. Weilhammer ${ }^{j}$, J. Werner ${ }^{\text {w }}$, A.M. Wetherell ${ }^{\text {j, J.H. Wickens }}{ }^{\text {ad }}$, J. Wikne ${ }^{\text {al }}$, G.R. Wilkinson s, W.S.C. Williams s, M. Winter ${ }^{\text {ab }}$, D. Wormald ${ }^{\text {al }}$, G. Wormser ${ }^{\mathrm{p}}$, K. Woschnagg ${ }^{\mathrm{ai}}$, N. Yamdagni ${ }^{\circ}$, P. Yepes ${ }^{\mathrm{i}}$, A. Zaitsev ${ }^{\mathrm{z}}$, A. Zalewska ${ }^{\text {at }}$, P. Zalewski ${ }^{\mathrm{p}}$, D. Zavrtanik ${ }^{\text {, }}$, E. Zevgolatakos ${ }^{\text {aa }}$, G. Zhang ${ }^{\text {w }}$, N.I. Zimin ${ }^{\mathrm{e}}$, M. Zito ag, R. Zuberi s, R. Zukanovich Funchal ${ }^{y}$, G. Zumerle ${ }^{\text {ak }}$ and J. Zuniga ${ }^{\mathrm{g}}$

"LIP, IST, FOUL - Al: Elias Garcia, 14 - Ie, P-1000 Lishon Codex, Portugal an Institute of Nuclear Physics, N.C.S.R. Demokritos, P.O. Box 60228, GR-15310 Athens, Grecce

"Centre de Recherche Nucléaire, IN2P3-CNRS / ULP-BP20, F-67037 Strasbourg Cedex, France

"Dipartimento di Fisica, Uni ersità di Bologna and INFN, Via Imerio 46, I-40126 Bologna, Italy

"ad Physics Department, Unil: Instelling Antwerpen, Unicersiteitsplein I, B-2610 Wilrijk, Belgium and IIHE, ULB-VUB, Pleinlaan 2, B-1050 Brussels, Belgium and Faculté des Sciences, Unit. de I'Etat Mons, Ai: Maistriau 19, B-7000 Mons, Belgium "Dipartimento di Fisica Sperimentale, Unicersità di Torino and INFN, Via P. Giuria I, 
"I Dipartimento di Fisica, Università di Roma II and INFN, Tor Vergata, I-00173 Rome, Italy

"Centre d'Etude de Saclay, DSM/DAPNIA, F-91191 Gif-sur-Ylette Cedex, France

"Wh Department of Physics, Unitersity of Liverpool, P.O. Box 147,GB-Licerpool L69 3BX, UK

"Department of Radiation Sciences, Unicersity of Uppsala, P.O. Box 535, S-75I 2I Uppsala, Sweden

"ij Dipartimento di Fisica, Unitersità di Genoa and INFN, Via Dodecaneso 33, 1-16146 Genoa, Italy

"k Dipartimento di Fisica, Unicersità di Padola and INFN, Via Marzolo 8, I-35131 Padua, Italy "Physics Department, Unicersity of Oslo, Blindern, N-l000 Oslo 3, Norway

${ }^{a m}$ Facultad de Ciencias, Unicersidad de Santander, al, de los Castros, E-39005 Santander, Spain

"Ames Laboratory and Department of Physics, Iowa State University, Ames 1A 50011, USA "No Niels Bohr Institute, Blegdamsiej 17, DK-2100 Copenhagen 0, Denmark

"Inst. Nuclear Studies and, Unitersity of Warsaw, Ul. Hoza 69, PL-0068I Warsaw, Poland

"National Technical Unicersity, Physics Department, Zografou Campus, GR-15773 Athens, Greece

"Unix: d'Aix - Marseille II - Case 907 - 70, route Léon Lachamp, F-13288 Marseille Cedex 09, France

"Institut "Jozef Stefan", Ljubliana, Slotenia

"' High Energy Physics Laboratory, Institute of Nuclear Physics, Ul. Kawiory 26 a, PL-30055 Krakow 30, Poland

"un School of Physics and Materials, University of Lancaster - Lancaster LA1 4YB, UK

"Centro Brasileiro de Pesquisas Físicas, rua Xacier Sigaud 150, RJ-22290 Rio de Janeiro, Brazil

${ }^{b}$ Institut für Hochenergiephysik, Österr. Akad. d. Wissensch., Nikolsdorfergasse 18, A-1050 Vienna, Austria 'Rutherford Appleton Laboratory, Chilton, GB - Didcot OXII OQX, UK

"NIKHEF-H, Postbus 41882, NL-1009 DB Amsterdam, The Netherlands

'Joint Institute for Nuclear Research, Dubna, Head Post Office, P.O. Box 79, 101000 Moscow, USSR

'Research Institute for High Energy Physics, SEFT, Siltaluorenpenger $20 \mathrm{C}, \mathrm{SF}-00170 \mathrm{Helsinki, \text {Finland }}$ "IFIC, Valencia-CSIC, and D.F.A.M.N., U. de Valencia, At da. Dr. Moliner 50, E-46100 Burjassot, Valencia, Spain

${ }^{h}$ Department of Physics, University of Lund, Söl egatan 14, S-22363 Lund, Sweden

' Department of Physics, University of Bergen, Allégaten 55, N-5007 Bergen, Norway 'CERN, CH-1211 Genera 23, Switzerland

${ }^{k}$ Physics Laboratory, Unicersity of Athens, Solonos Str. 104, GR-10680 Athens, Greece

'Dipartimento di Fisica, Unicersità di Milano and INFN, Via Celoria 16, I-20133 Milan, Italy

${ }^{m}$ Université Claude Bernard de Lyon, 43 Bd du 11 Novembre 1918, F-69622 Villeurbanne Cedex, France

"Institut für Experimentelle Kermphysik, Unicersität Karlsruhe, Postfach 6980. D.7500 Karlsruhe 1, Germany

"Institute of Physics, University of Stockholm, Vanadist ägen 9, S-11346 Stockholm, Sweden

"Unicersité de Paris-Sud, Lab. de l'Accélérateur Linéaire. Bat 200, F-91405 Orsay, France

"Institut des Sciences Nucléaires, Unicersité de Grenoble l, F-38026 Grenoble, France

r Dipartimento di Fisica, Unicersità di Trieste and INFN, Via A. Valerio 2, 1-34127 Trieste, Italy and Istituto di Fisica, Università di Udine, I-33100 Udine, Italy

${ }^{s}$ Nuclear Physics Laboraton, Unicersity of Oxford, Keble Road, GB - Oxford OXI 3RH, UK

' Istituto Superiore di Sanità, Ist. Naz. di Fisica Nucl. (INFN), Viale Regina Elena 299, I-0016I Rome, Italy

"Unicersidad Complutense, Aida. Complutense s / n, E-28040 Madrid, Spain

'LPNHE, Unicersités Paris VI et VII, Tour 33 (RdC), 4 place Jussieu, F-75230 Paris Cedex 05, France

"Fachbereich Physik, Unicersity of Wuppertal, Postfach 100 127, D-5600 Wuppertal 1, Germany

${ }^{x}$ Depto. de Fisica, Pontificia Unit. Católica, C.P. $3807 I$ RJ-22453 Rio de Janeiro, Brazil

"College de France, Lab. de Physique Corpusculaire, 11 pl. M. Berthelot, F-75231 Paris Cedex 05, France

${ }^{z}$ Inst. for High Energy Physics, Serpukow P.O. Box 35, Protino (Moscow Region), Russian Federation

Received 24 July 1992

Revised 9 September 1992

Accepted for publication 9 September 1992

An analysis of the fluctuations in the phase space distribution of hadrons produced in the decay of $78829 \mathrm{Z}^{0}$ has been carried out, using the method of factorial moments. The high 
statistics collected by the DELPHI experiment at LEP during 1990 allowed studies of the event sample both globally and in intervals of $p_{1}$ and multiplicity, and for different jet topologies and for single jets. A large contribution to the factorial moments of the one-dimensional data on rapidity with respect to the event axis comes from hard gluons. Details of factorial moments in two and three dimensions are presented. Influences of resonance decays have been studied by Monte Carlo simulation: one-dimensional factorial moments at low $p_{1}$, and two-, three-dimensional analyses are affected. Parton shower models describe the data reasonably well.

\section{Introduction}

This paper presents results of the study of fluctuations in the distribution of hadrons produced in $\mathrm{e}^{+} \mathrm{e}^{-}$collisions at the $\mathrm{Z}^{0}$ energy, using the DELPHI detector at LEP. It follows and complements previous studies [1] using a sample of hadronic events with 10 times higher statistics. Preliminary results have already been reported [2].

In order to provide a quantitative test of anomalous multiplicity fluctuations (spikes) in variables intervals of rapidity, Bialas and Peschanski [3] proposed in 1986 to analyze the distributions of multiplicity in terms of normalized factorial moments. Given an experimental distribution of particles in the rapidity interval from $-Y / 2$ to $Y / 2$, the interval $Y$ is divided into $M$ equal subintervals, each of size $\delta y=Y / M$. If $N$ is the number of particles in the whole rapidity interval and $n_{m}$ the number of particles in the $m$ th bin $(m=1, \ldots, M)$, the factorial moment of (integer) rank $j$ of the distribution is defined by

$$
F_{j}(\delta y)=\frac{M^{j-1}}{\langle N\rangle^{j}}\left\langle\sum_{m=1}^{M} n_{m}\left(n_{m}-1\right) \ldots\left(n_{m}-j+1\right)\right\rangle,
$$

where the averages are taken over many events. The factorial moment of rank $j$ for a rapidity interval $\delta y$ selects events with $j$ particles or more in at least one bin and is sensitive to events with density fluctuations in rapidity.

Simple models representing the hadronization process as a random cascade with self-similar structure predict a powerlike increase of the factorial moments when the bin size $\delta y$ approaches zero, i.e.

$$
F_{j} \propto(1 / \delta y)^{f_{j}}, \quad f_{j}>0
$$

and the validity of the above relation was taken by the authors of ref. [3] as the definition of intermittency, a term borrowed from hydrodynamics, as are most of the mathematical techniques used in this field [4]. Relation (2) has been taken in this paper as definition of intermittency, as no universally accepted definition exists in the literature.

The first direct measurement of factorial moments in $\mathrm{e}^{+} \mathrm{e}^{-}$annihilations by the TASSO collaboration [5], at a centre-of-mass energy of around $35 \mathrm{GeV}$, appeared 
to show an intermittency effect that could not be explained by the JETSET Parton Shower Monte Carlo [6] (JETSET PS in the following), nor by the MarchesiniWebber [7] or Hoyer [8] models. The TASSO results agreed with an indirect analysis of the HRS data [9] at $\sqrt{s}=29 \mathrm{GeV}$. Later, the HRS collaboration has made a further analysis [10], which agreed with ref. [9], but no explicit comparison with Monte Carlo models was made. The predictions of various models for $\mathrm{e}^{+} \mathrm{e}^{-}$ interactions differ considerably at high energies [11]. This situation has motivated an investigation of possible intermittency effects in $\mathrm{e}^{+} \mathrm{e}^{-}$annihilation at the $\mathrm{Z}^{0}$ energy. An initial analysis [1] used the earliest data taken by DELPHI [12] at the $\mathrm{e}^{+} \mathrm{e}^{-}$storage ring LEP. The presence of intermittency, as defined in eq. (2), was unclear when just the rapidity distribution of final-state hadrons was studied, but evident in a two-dimensional analysis. In all cases the results were compatible with the predictions of parton shower models [13]. Subsequently, CELLO [14] reported an agreement with parton shower models at the same energy as TASSO, OPAL [15], ALEPH [16] and L3 [36] at the same energy as DELPHI.

The subject of intermittency has motivated many theoretical studies and experimental investigations over the last few years, for which exhaustive reviews have been published [17]. These often introduce new physics to explain the phenomenon. However many authors have pointed out that self-similar cascading mechanisms $[3,18,19]$, or models in which simple hypotheses for standard shortrange correlations are introduced [20], can also reproduce the observed effects.

\section{Event selection}

The sample of events used in the present analysis was collected by the DELPHI detector at the LEP $\mathrm{e}^{+} \mathrm{e}^{-}$collider during 1990. A description of the DELPHI detector can be found elsewhere [12]. Only charged particles reconstructed by the central detectors were used in this analysis, selected if their:

(a) polar angle $\theta$ with respect to the beam axis was between $25^{\circ}$ and $155^{\circ}$;

(b) momentum $p$ was between 0.1 and $20 \mathrm{GeV} / c$;

(c) track length in the Time Projection Chamber (TPC) was over $50 \mathrm{~cm}$;

(d) projection of impact parameter with respect to the origin in the plane perpendicular to the beam axis was below $5 \mathrm{~cm}$

(e) impact parameter along the beam with respect to the origin was below $10 \mathrm{~cm}$. Hadronic events were selected by requiring:

$(\alpha)$ at least 5 charged particles with momentum $p$ above $0.2 \mathrm{GeV} / c$;

$(\beta)$ a total energy detected in charged particles (assuming $\pi^{ \pm}$mass) above 15 $\mathrm{GeV}$;

$(\gamma)$ a total energy in charged particles above $3 \mathrm{GeV}$ in each of the two hemispheres with respect to the beam axis, i.e. $\cos \theta<0$ and $\cos \theta>0$; 
( $\delta$ ) a sphericity axis with polar angle between $40^{\circ}$ and $140^{\circ}$;

( $\epsilon$ ) total momentum imbalance below $30 \mathrm{GeV} / c$;

A sample of 78829 events satisfied these cuts. Beam-gas scatters, $\gamma \gamma$ interactions and decays of the $\mathrm{Z}^{0}$ into $\tau^{+} \tau^{-}$constitute less than $0.3 \%$ of the selected sample.

The Monte Carlo simulation program DELSIM [21] was used to correct the data for the geometrical acceptance, kinematical cuts, resolution, particle interactions with the detector material and other detector imperfections. A sample of $Z^{0}$ decays, with similar statistics to the sample of real events, (see below) was generated with JETSET 7.2 PS [6] and followed through this detailed simulation of the detector. The generated event sample contained all final-state charged particles with a lifetime above $10^{-9} \mathrm{~s}$ before any tracking was done through the detector. All particles were then followed through the DELPHI detector, including all the effects of decays and interactions, in order to simulate the raw hits in the sensitive volumes. These data were then processed through the same reconstruction and analysis chain as the real data to give the final accepted event sample. From the samples of accepted and generated events, correction factors

$$
C(\delta y)=\frac{F(\delta y)_{\text {generated }}}{F(\delta y)_{\text {accepted }}}
$$

were computed. These factors were then used to correct the quantities calculated from the real data and are shown above the figures.

This analysis uses the rapidity defined as $y=\frac{1}{2} \ln \left[\left(E+p_{\ell}\right) /\left(E-p_{\ell}\right)\right]$ where $E$ is the charged particle energy assuming the pion mass and $p_{\ell}$ its longitudinal momentum, transverse momentum $p_{\mathrm{t}}$ and azimuthal angle $\phi$ (see subsect. 3.2). All three variables, where not explicitly stated, are defined with respect to the sphericity axis.

The resolving power of the detector was estimated by the Monte Carlo simulation. Typically 2, 3, 4, 5 tracks could be resolved if their rapidities differed by more than $0.04,0.06,0.07,0.08$, which are less than the smallest rapidity interval used in the experimental studies here presented. Resolutions on $p_{\mathrm{t}}$ and $\phi$ variables were found to be lower than the smallest bins used in the analysis.

A possible bias in the evaluation of the "true" factorial moments due to limited statistics has been investigated by dividing the data into 10 equally populated subsamples. Factorial moments of rank $q$ were calculated for each of the 10 subsamples and their average value was computed; the comparison between this value and that calculated for the whole sample gives the estimate of bias. Monte Carlo samples (JETSET PS at generator level) and real data samples of different sizes have been used and in both cases it was found that the bias is negligible for samples of 10000 events or more, up to the 5th rank. Moreover, as the analysis 
uses similar statistics for the simulated and real data, such effects should cancel in any comparison.

A correction to the $\gamma$ conversion length in the detector has been included. This correction was estimated using the final simulated PS events and adding extra particles (electrons and positrons) produced by $\gamma$ generated by JETSET Monte Carlo and then converted in an extra $1.1 \%$ of a radiation length in the Inner Detector; comparison between factorial moments obtained with and without applying this procedure gives a quantitative evaluation of the systematic shift.

\section{Analysis and results}

In this section factorial moments of projections of distributions of charged hadron from the decay of the $Z^{0}$ are compared with the predictions of QCD-based Monte Carlo programs.

Initially the corrected data were compared with JETSET PS with default parameters, as these reproduce the hadronic final states from the decay of the $\mathrm{Z}^{0}$ satisfactorily, both for shape variables [22-24] and, more importantly, for total multiplicity and for multiplicity in restricted intervals of rapidity and in different jet topologies [25]. They are also compared with JETSET PS after retuning of the parameters $[23,26]$; with the JETSET 7.2 Monte Carlo with a matrix-element calculation up to $\mathrm{O}\left(\alpha_{\mathrm{s}}^{2}\right)$ with optimized parameters [23] (JETSET ME retuned) and with the ARIADNE [27] Monte Carlo using optimized parameters [23,26].

Taking the uncertainties in the retuned parameters into account, the different retuned versions of JETSET PS and ARIADNE give compatible results on factorial moments. The figures show results for JETSET PS and ARIADNE retuned as in ref. [23].

\subsection{ANALYSIS OF ONE-DIMENSIONAL FACTORIAL MOMENTS OF RAPIDITY}

Fig. 1 shows the corrected factorial moments of the rapidity distribution between -2 and +2 . The logarithms of the factorial moments grow with the logarithm of the number of subdivisions for all ranks, but this growth is not linear. The figure also shows the predictions for JETSET PS default, JETSET PS retuned, JETSET ME retuned and ARIADNE. The hatched regions represent the uncertainties in the moments for the models with retuned parameters except for JETSET ME.

The JETSET PS default represents the data well, as do the retuned JETSET PS and retuned ARIADNE models. In JETSET ME the retuning of the fragmentation parameters causes a big change both in the magnitude of the factorial moments and in the slope of their distribution for small values of $\delta y$, as was 

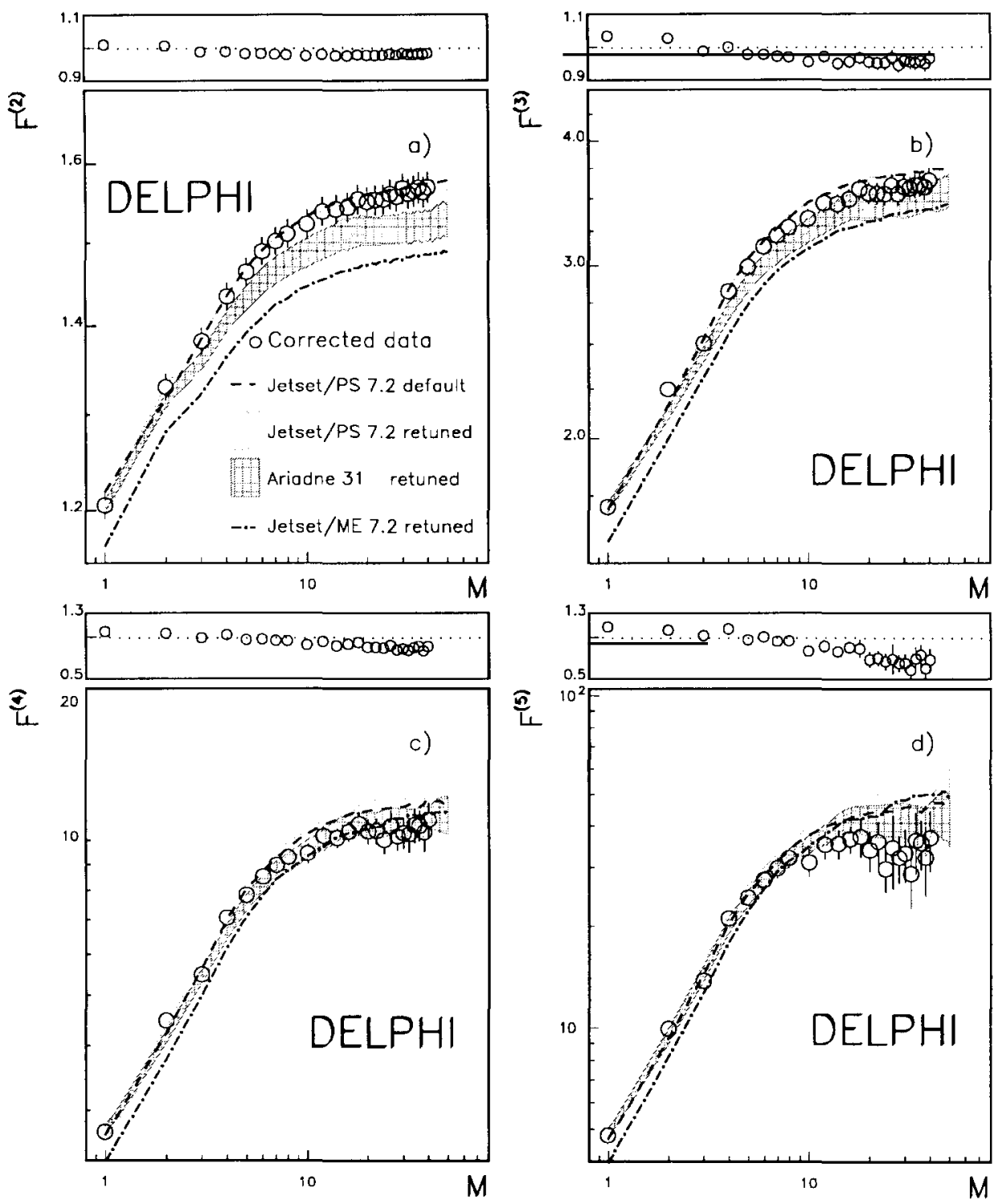

Fig. 1. Bi-logarithmic plots of factorial moments of rank 2 (a), 3 (b), 4 (c) and 5 (d) versus the number $M$ of subdivisions of the rapidity interval $(-2,+2)$, compared with several Monte Carlo predictions. Hatched areas are introduced for models in which retuned parameters are quoted with errors. Correction factors are shown in the upper small plots.

previously shown [1]. However, even after retuning, the ME model fails to reproduce the data quantitatively.

In this analysis (fig. 1) the central region of the rapidity interval was chosen where the density of particles is approximately uniform, because a non-uniform 
population can fake the signal from the anomalous clusters [28]. However, this excludes the particles closer to the core of the jets. To overcome this Bialas and Gazdzicki, and Ochs [29] suggested using a variable $\tilde{y}$, that is the $y$ distribution rebinned in the $y_{\min }, y_{\max }$ interval in such a way that population is uniform on average

$$
\begin{gathered}
\tilde{y}(y)=\frac{1}{C} \int_{y_{\min }}^{y} \frac{1}{N_{\mathrm{evt}}} \frac{\mathrm{d} n}{\mathrm{~d} y}\left(y^{\prime}\right) \mathrm{d} y^{\prime}, \\
C=\int_{y_{\min }}^{y_{\max }} \frac{1}{N_{\mathrm{evt}}} \frac{\mathrm{d} n}{\mathrm{~d} y}\left(y^{\prime}\right) \mathrm{d} y^{\prime},
\end{gathered}
$$

where $\left(1 / N_{\text {evt }}\right) \mathrm{d} n(y) / \mathrm{d} y$ is the rapidity distribution. The $\tilde{y}$ range is 0 to 1 . Using self-similar models for the hadronization these authors found that factorial moments defined with respect to $\tilde{y}$ predict the power law (2) even more closely than when using $y$.

Factorial moments in the $\tilde{y}$ distribution corresponding to the $y$ region between -5 and +5 gave results qualitatively similar to those using the $y$ distribution.

In the following, unless otherwise stated, the variable $\tilde{y}$ is used when analyzing the dependence of the factorial moments on $p_{t}$, the multiplicity and the jet topology and $y$ is taken between -5 and +5 . This provides a convenient way to compare the results, independent of changes in the selected $y$ distribution caused by the kinematic selections.

3.1.1. Dependence on $p_{t}$. The NA22 collaboration, studying $\pi^{+} \mathrm{p}$ and $\mathrm{K}^{+} \mathrm{p}$ collision at $250 \mathrm{GeV} / c$, reported a striking disagreement between data and hadronic Monte Carlo models for charged particles at low $p_{t}$ [30].

Different dynamics in hadronic and $\mathrm{e}^{+} \mathrm{e}^{-}$collisions influence the $p_{1}$ distribution. Nevertheless it is interesting to see whether the $p_{\mathrm{t}}$ distribution in the data is populated in the same way as JETSET PS. Moreover, the low- $p_{\mathrm{t}}$ region is almost free from effects related to hard gluon radiation, which significantly affect the factorial moments [16].

Charged particles were divided into three regions of $p_{\mathrm{t}}\left(p_{\mathrm{t}}<0.255,0.255<p_{\mathrm{t}}<\right.$ $\left.0.532,0.532<p_{\mathrm{t}}<2 \mathrm{GeV} / c\right)$ chosen so that the total number of particles in each is the same, in order to avoid any bias in the result from statistical differences. After dividing the final-state particles into these slices of $p_{\mathrm{t}}$ the variable $y$ was transformed into $\tilde{y}$ for each slice, as discussed above.

Fig. 2 shows that the data are well reproduced by parton shower models in these three $p_{\mathrm{t}}$ regions.

At the lowest $p_{\mathrm{t}}$ the factorial moments follow a power-law behaviour for $M$ above 5 but Monte Carlo studies show that it is due to resonance decays; see subsect. 3.3 for a general discussion.

The highest $p_{\mathrm{t}}$ region shows higher factorial moments, but they saturate for large $M$. This behaviour is compatible with a rise due to the spikes caused by the 

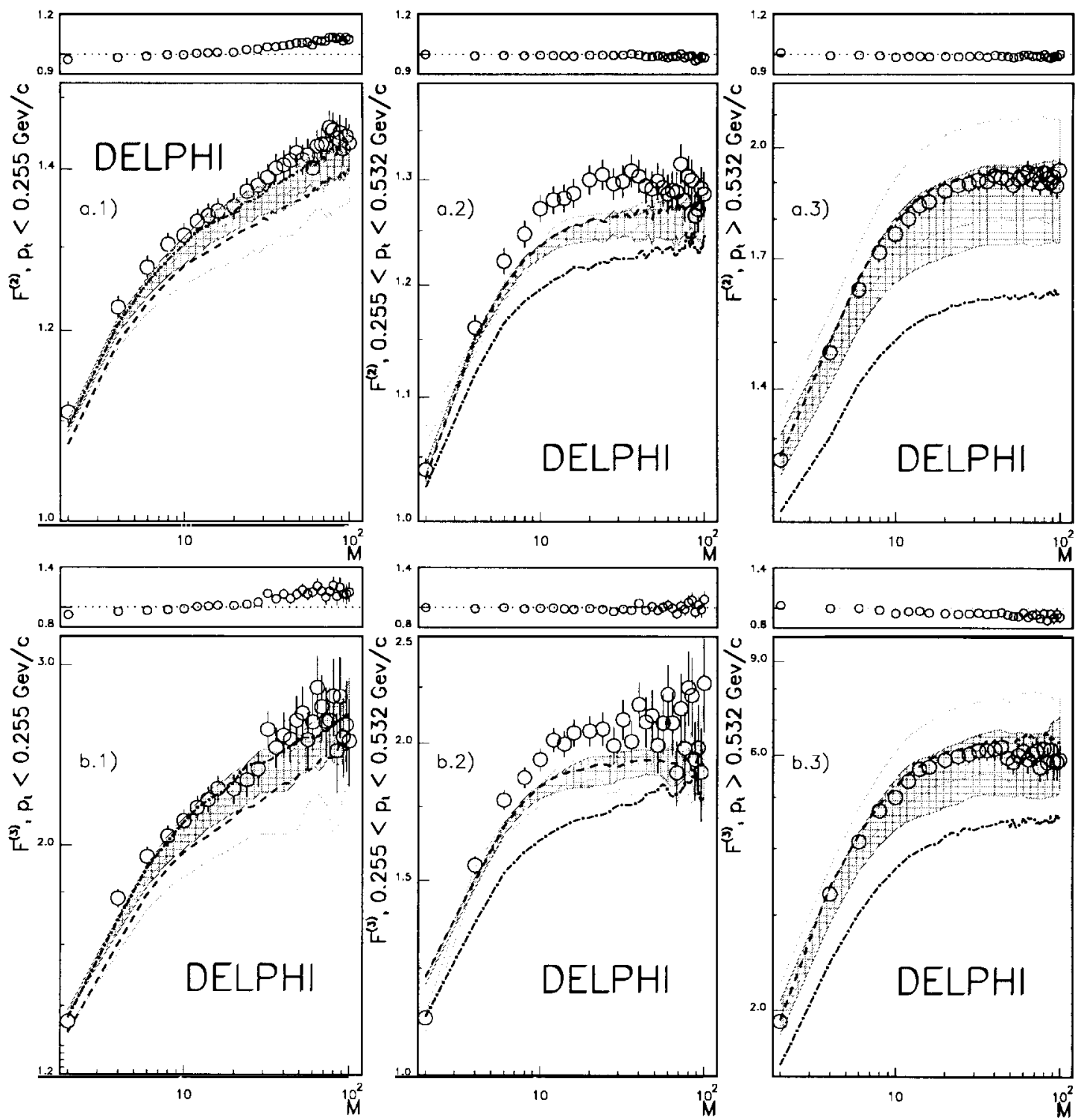

Fig. 2. Factorial moments of the $\tilde{y}(y)$ distribution ( $y$ between -5 and +5 ) of rank 2 (a) and 3 (b) for three $p_{\mathrm{t}}$ intervals versus the number $M$ of subdivisions; bi-logarithmic scale is used. DELPHI corrected data (white circles) are compared with several models as in fig. 1. Correction factors are shown above each plot.

presence of the hard gluon jets, and flattening off when fluctuations are reduced because $\delta y$ becomes of the same order or smaller than the third jet size. For small $M$-values factorial moments are sensitive to the large-scale event structure, where 

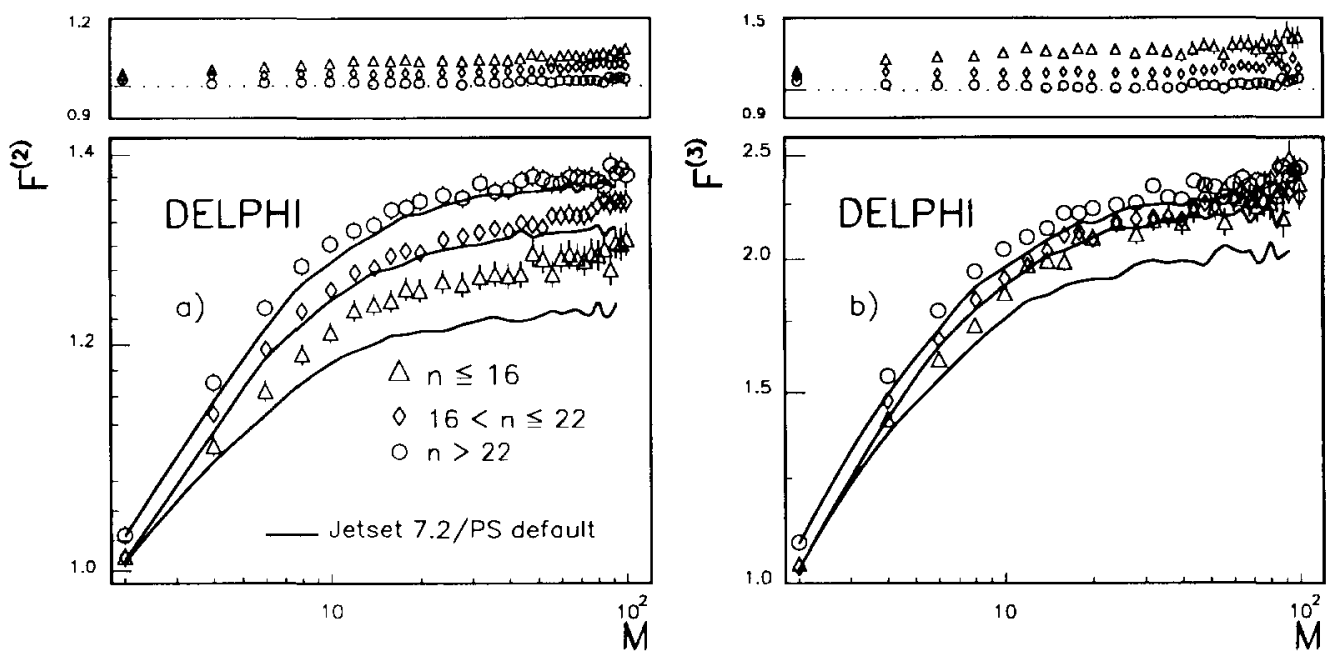

Fig. 3. Factorial moments of the $\tilde{y}$-distribution ( $y$ between -5 and +5 ) of rank 2 (a) and 3 (b) for different observed multiplicity intervals. DELPHI corrected data compared with JETSET 7.2 PS Monte Carlo predictions (see text). Correction factors are shown in the upper small plots.

any jet not collinear with the event axis behaves as a "spike"; whereas at large $M$ the values are sensitive to the structure inside the jets.

The intermediate slice in $p_{\mathrm{t}}$ has lower factorial moments which show saturation for large $M$.

3.1.2. Dependence on charge multiplicity. The behavior of factorial moments has been studied as a function of the charged multiplicity of the event.

The sample of hadronic events has been divided in 3 sub-samples with reconstructed charged particle multiplicities $n$ in the intervals $n \leqslant 16,16<n \leqslant 22$ and $n>22$ in the rapidity interval from -5 up to +5 ; they correspond respectively to the low-multiplicity tail, to the central region around the maximum and to the high-multiplicity tail in the multiplicity distribution and contain more than 10000 hadronic events each. The $\tilde{y}$-variable has been calculated for each of these regions.

The results shown in fig. 3 demonstrate that the factorial moments rise with multiplicity.

To compare data with Monte Carlo models one faces the problem of associating an observed multiplicity to a generated event. To do this the matrix $M(m, n)$ has been estimated, whose elements are the probability of an event with observed charged multiplicity $n$ to have a true charged multiplicity $m$. The three generated sub-samples are then constructed by weighting each event by the proper factor $M(m, n)$ according to its multiplicities ( $m$-generated, $n$-observed). The matrix has been estimated using the JETSET 7.2 PS + DELSIM events sample. The $M(m, n)$ values were also computed using Herwig [31] + DELSIM program and the differences were found to be less than the statistical errors on average.

Fig. 3 shows the predictions of the JETSET 7.2 PS Monte Carlo with default 

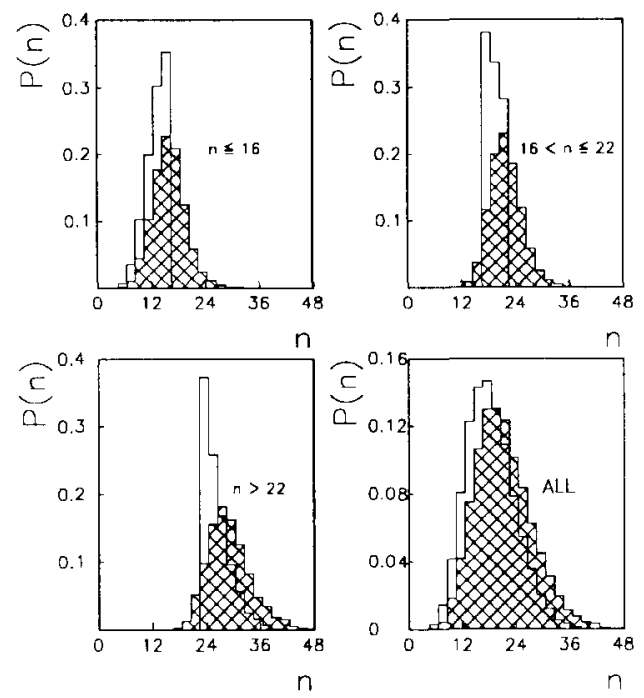

Fig. 4. Event multiplicity distributions at generation level (hatch histograms) and after detector simulation and reconstruction (plain histograms) for the three observed event multiplicity samples and for the full sample.

settings. At low multiplicities differences between data and Monte Carlo are of the order of three standard deviations, for medium multiplicities of two and for high multiplicities less than one. However this disagreement is about the same size as the shifts in the JETSET results when retuned parameters are used in figs. 1 and 2 . The shift due to the correction for the extra $1.1 \%$ radiation length in the detector is not included here, see sect. 2 .

The dependence of factorial moments on multiplicity is stronger before the correction to the real data, which can be seen from the correction factor behaviours for the three multiplicity regions. In particular for the low and the medium intervals, factorial moments for corrected data or Monte Carlo samples are considerably higher than for uncorrected ones (or Monte Carlo after detector simulation and reconstruction), mainly because multiplicity distributions for the former samples are higher and much broader (with tails) than the latter ones. In contrast, this difference is not observed for the full samples where the two distributions are very similar (fig. 4).

A similar analysis was also performed by using $y$ in the interval from -2 to 2 and slicing the hadronic event sample in regions of observed charged particle multiplicities in the plateau of $n \leqslant 10,10<n \leqslant 15$ and $n>15$. The results are qualitatively the same.

3.1.3. Dependence on jet topology. The two-jet and the three-jet events were selected using the JADE/E0 [32] clustering algorithm, with values of 0.04 and 0.01 of the resolution parameter $y_{\text {cut }}$, and using the following additional selections for 

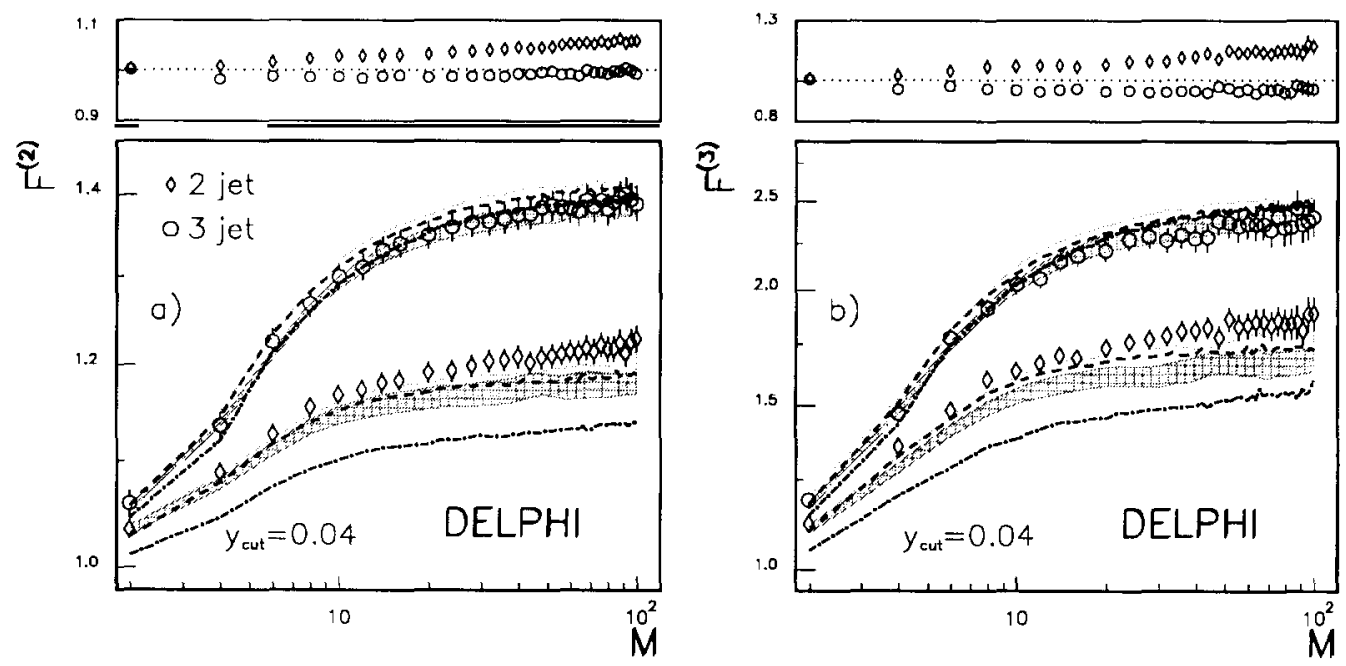

Fig. 5. Factorial moments of rank 2 (a) and 3 (b) of the $\tilde{y}$ ( $y$ between -5 and +5 ) distribution for two-jet and three-jet events $\left(y_{\text {cut }}=0.04\right)$ versus the number $M$ of subdivisions; bi-logarithmic scale is used. DELPHI corrected data (open symbols) compared with models as in fig. 1. Correction factors are shown above each plot.

cleaner samples of two-jet and three-jet events. Each jet must contain at least 3 charged particles. The axis of each jet was then defined as the direction of the sum of the momenta of the charged particles in the jet. This axis had to be in the region of polar angle between $40^{\circ}$ and $140^{\circ}$. The estimated jet energies are based on the angles between the jets, assuming massless kinematics at an energy equal to the $Z^{0}$ mass. Both the estimated jet energy and the sum of the observed charged particle energies must be greater than $5 \mathrm{GeV}$.

The two-jet events must have over $170^{\circ}$ between the two jet axes and each jet axis within $8^{\circ}$ of the sphericity axis. For the three-jet events, the three jets are required to be planar with the sum of the angles between their axes greater than $355^{\circ}$.

These selections left 15044 two-jet and 14843 three-jet events for $y_{\text {cut }}=0.01$ and 31640 two-jet, 12749 three-jet events for $y_{\text {cut }}=0.04$.

Fig. 5 shows results for $y_{\text {cut }}=0.04$ and fig. 6 for $y_{\text {cut }}=0.01$. No striking disagreement with respect to JETSET PS is observed.

Factorial moments are always larger in three-jet than in two-jet events. This is compatible with spikes in rapidity caused by hard gluon jets, as was found in the $p_{t}$ analysis of subsect. 3.1.1. This means that any study of intermittency which is to be free of effects from hard radiation needs to analyse two-jet events or to calculate factorial moments for each jet in an event. In this way the core of each jet is analysed to look for particle density spikes that are not from hard QCD effects. 

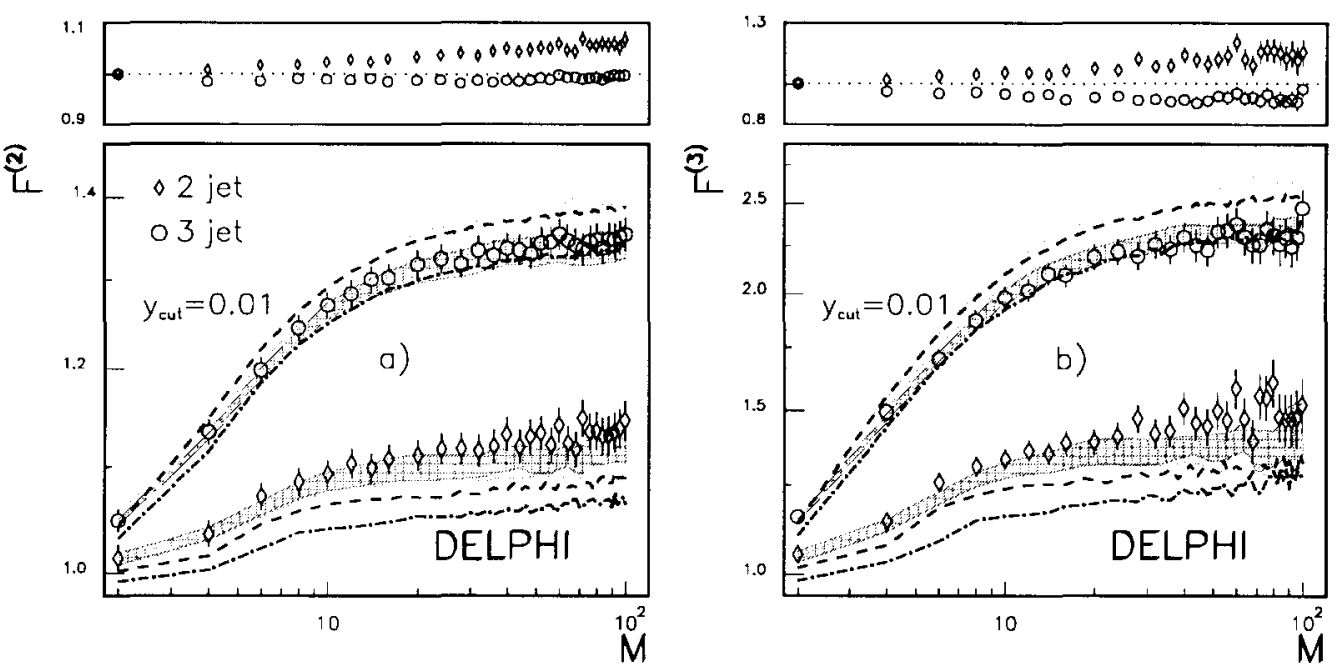

Fig. 6. As in fig. 5 but with $y_{\text {cut }}=0.01$.

Hence in three-jet events, selected from the JADE/E0 algorithm using $y_{\text {cut }}=$ 0.04 , factorial moments in $\tilde{y}$ were also calculated for the charged particles belonging to jet 1 , jet 2 and jet 3 , ordered by energy, defining the rapidity with respect to the jet axis.

The results are plotted in fig. 7 for data and Monte Carlo (only JETSET PS retuned). The behaviour of the factorial moments is different from fig. 5. The first
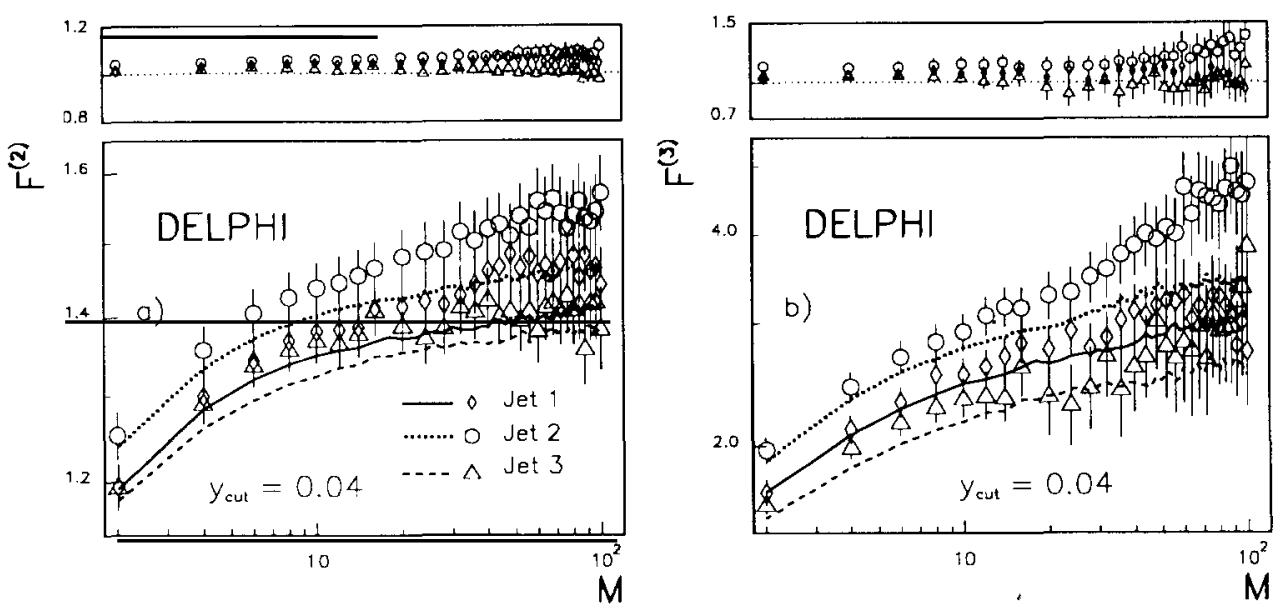

Fig. 7. The factorial moments of rank 2 (a) and 3 (b) of the first, second and third jet ordered by their energy. The $\tilde{y}$ ( $y$ between -1 and +5 ) distribution is used. $M$ is the number of subdivisions; bi-logarithmic scale is used. DELPHI corrected data (open symbols) are compared with JETSET 7.2 PS Monte Carlo predictions with retuned settings (curves). Corrections factors are shown above each plot. 
and third jet have similar behaviour, the second displays instead higher factorial moments.

\subsection{PROJECTIONS ONTO HIGHER DIMENSIONS}

Recent theoretical work [35] suggests that the saturation of factorial moments at $\delta y \simeq 0.1$ is the result of the smearing of fluctuations due to the projection of phase space onto a one-dimensional subspace. Stronger intermittency effects should appear in two and three dimensions. The variables used here for two- and three-dimensions factorial moments are $y, \phi$ and $p_{\mathrm{t}}$. The angle $\phi$ is measured starting from the azimuth $\phi_{\mathrm{s} 2}$ of the second eigenvector of the momentum tensor, because the $\phi$ distribution is peaked at $\phi_{\mathrm{s} 2}$.

When increasing the number of dimensions, the problem of the non-uniformity of the distributions becomes of primary importance. The variables $y, p_{\mathrm{t}}$ and $\phi$ are not uniformly distributed within each event and are correlated with each other and therefore they have been transformed in the way suggested by Bialas and Gazdzicki [29]. This transformation acts in multi-dimensional spaces as $\tilde{y}$ does in one dimension. In two dimensions it defines two new variables $X_{2}(y), Y_{2}(y, \phi)$ with uniform mean population of the $\left(X_{2}, Y_{2}\right)$ space. This is often called the $(\widetilde{y, \phi})$ transform. In three dimensions $\left(\left(y, \phi p_{\mathrm{t}}\right)\right.$ transform $)$ it defines $X_{3}(y)$, $Y_{3}(y, \phi), Z_{3}\left(y, \phi, p_{\mathrm{t}}\right)$, where $X_{2}=X_{3}=\tilde{y}$ and $Y_{2}=Y_{3}$. The $X, Y$ and $Z$ range is 0 to $1 ; p_{\mathrm{t}}$ were selected in the range 0 to $2 \mathrm{GeV} / c, \phi$ between 0 and $2 \pi$ and $y$ between -5 and +5 .

To calculate factorial moments the phase space has been subdivided by halving the bins first for $X$, then $Y$ and $Z$ intervals; then again $X, Y$ and $Z$ and so on. If $d$ is the dimension of the phase space, when $M=2^{d n}$ each single variable interval has been divided in $M^{\prime}=2^{n}$ bins. By plotting $F_{q}$ versus $\log _{2}(M) / d$ a vertical slice has always the same number of subdivisions in $X$ for all analyses and in $Y$ for two-dimensional and three-dimensional analyses.

Factorial moments have been calculated also for $\tilde{p}_{\mathrm{t}}$ and $\tilde{\phi}$ one-dimensional and $\left(\widetilde{y, p_{\mathrm{t}}}\right),\left(\widetilde{\phi, p_{\mathrm{t}}}\right)$ two-dimensional distributions.

The factorial moments of one-dimensional, two-dimensional and three-dimensional projections are plotted in fig. 8 and tabulated in table 1.

When intermittency is investigated in three dimensions $\left(\overline{y, \phi, p_{\mathrm{t}}}\right)$ it follows relation (2) much more closely. The predictions of JETSET PS Monte Carlo model remain in good agreement with the data for one-, two- and three-dimensional analyses.

Factorial moments in three dimensions display a stronger intermittent behavior than in the one- and two-dimensional analyses, as qualitatively predicted by three-dimensional models [35]. The one-dimensional $\tilde{y}$ factorial moments saturate at large $M$, and a similar effect, though less pronounced, is seen in two dimen- 

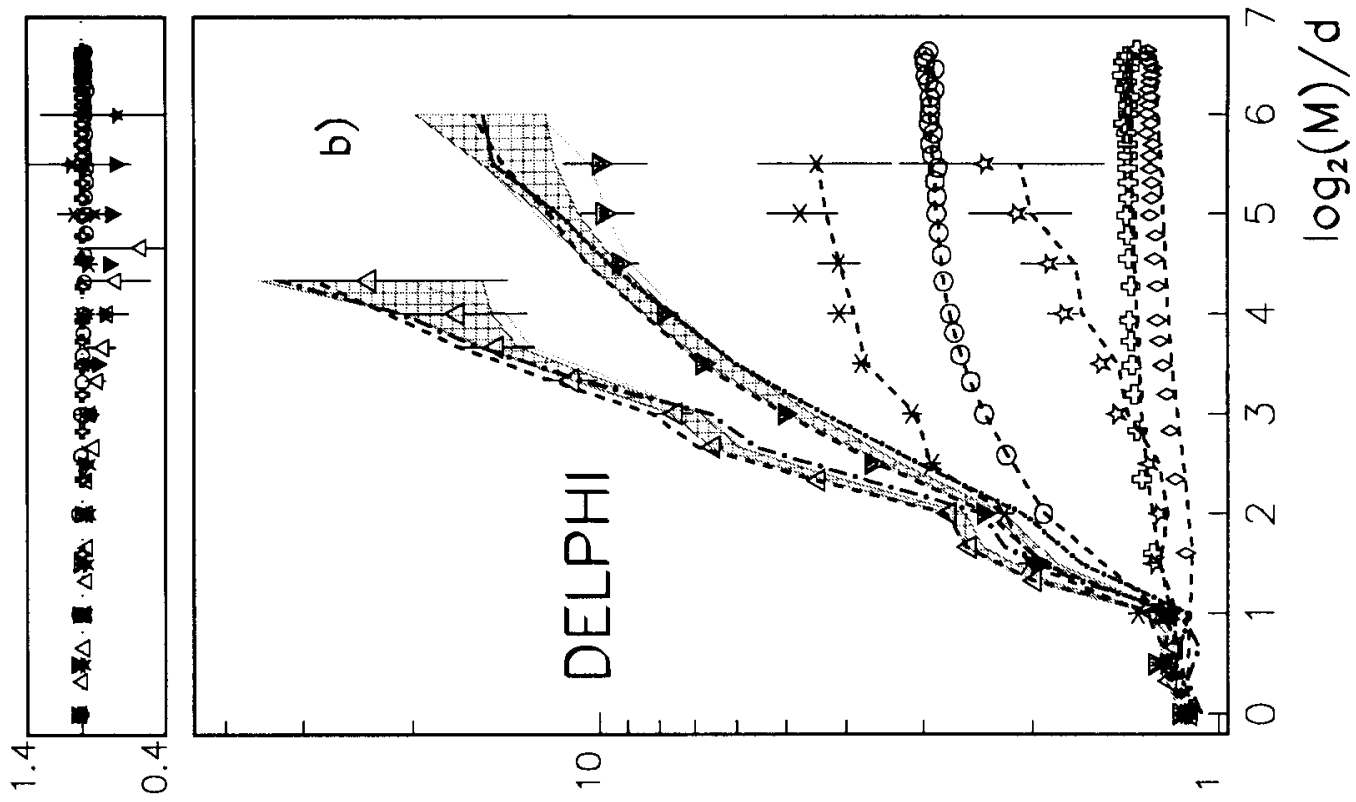

$\mid \begin{aligned} & 2 \cdot \frac{\pi}{3} \\ & 0\end{aligned}$

13

(c)

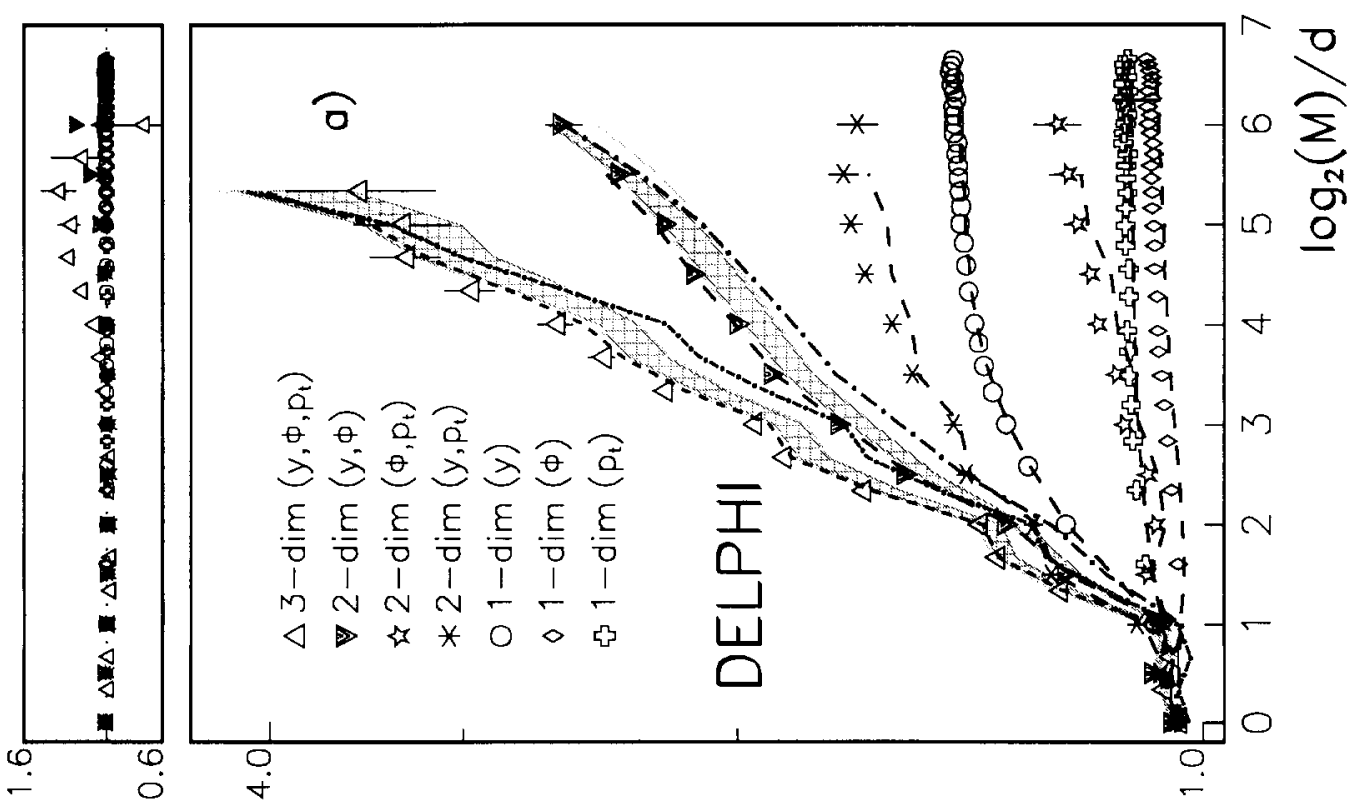


TABLE 1

Values of factorial moments for one-dimensional, two-dimensional and three-dimensional analyses shown in fig. 8

\begin{tabular}{|c|c|c|c|c|}
\hline \multirow{2}{*}{$\log _{2} \frac{M}{d}$} & \multicolumn{3}{|c|}{ Rank 2} & \\
\hline & $\tilde{y}$ & $\tilde{\phi}$ & $\underline{\tilde{p}_{\mathbf{t}}}$ & \\
\hline 1 & $1.076 \pm 0.007$ & $1.047 \pm 0.007$ & $1.040 \pm 0.007$ & \\
\hline 2 & $1.227 \pm 0.009$ & $1.039 \pm 0.007$ & $1.090 \pm 0.007$ & \\
\hline 3 & $1.341 \pm 0.01$ & $1.055 \pm 0.007$ & $1.110 \pm 0.007$ & \\
\hline 4 & $1.41 \pm 0.01$ & $1.071 \pm 0.007$ & $1.120 \pm 0.007$ & \\
\hline 5 & $1.44 \pm 0.01$ & $1.077 \pm 0.008$ & $1.123 \pm 0.008$ & \\
\hline 6 & $1.45 \pm 0.01$ & $1.086 \pm 0.009$ & $1.123 \pm 0.009$ & \\
\hline \multirow[t]{2}{*}{$\log _{2} \frac{M}{d}$} & \multicolumn{4}{|c|}{ Rank 3} \\
\hline & $\bar{y}$ & $\tilde{\phi}$ & $\tilde{p}_{\mathbf{1}}$ & \\
\hline 1 & $1.25 \pm 0.01$ & $1.15 \pm 0.01$ & $1.13 \pm 0.01$ & \\
\hline 2 & $1.91 \pm 0.02$ & $1.13 \pm 0.01$ & $1.28 \pm 0.01$ & \\
\hline 4 & $2.39 \pm 0.04$ & $1.20 \pm 0.01$ & $1.36 \pm 0.02$ & \\
\hline 4 & $2.72 \pm 0.05$ & $1.26 \pm 0.02$ & $1.40 \pm 0.02$ & \\
\hline 5 & $2.86 \pm 0.06$ & $1.29 \pm 0.02$ & $1.41 \pm 0.02$ & \\
\hline 6 & $3.00 \pm 0.07$ & $1.31 \pm 0.03$ & $1.40 \pm 0.03$ & \\
\hline \multirow{2}{*}{$\log _{2} \frac{M}{d}$} & \multicolumn{4}{|c|}{ Rank 2} \\
\hline & $(\widetilde{y, \phi})$ & $\left(\widetilde{\left.y, p_{\mathrm{t}}\right)}\right.$ & $\left(\widetilde{\phi, p_{\mathrm{t}}}\right)$ & $\left(\overline{\left.y, \phi, p_{t}\right)}\right.$ \\
\hline 0 & $1.047 \pm 0.007$ & $1.040 \pm 0.007$ & $1.042 \pm 0.007$ & $1.040 \pm 0.006$ \\
\hline 1 & $1.064 \pm 0.007$ & $1.066 \pm 0.007$ & $1.104 \pm 0.007$ & $1.082 \pm 0.007$ \\
\hline 2 & $1.337 \pm 0.01$ & $1.075 \pm 0.007$ & $1.288 \pm 0.009$ & $1.40 \pm 0.01$ \\
\hline 3 & $1.714+0.015$ & $1.126 \pm 0.009$ & $1.451 \pm 0.012$ & $1.96 \pm 0.02$ \\
\hline 4 & $1.99 \pm 0.02$ & $1.171 \pm 0.013$ & $1.588 \pm 0.017$ & $2.62 \pm 0.07$ \\
\hline 5 & $2.22 \pm 0.04$ & $1.208 \pm 0.023$ & $1.689 \pm 0.029$ & $3.29 \pm 0.24$ \\
\hline 6 & $2.59 \pm 0.07$ & $1.244 \pm 0.044$ & $1.673 \pm 0.051$ & \\
\hline \multirow[t]{2}{*}{$\log _{2} \frac{M}{d}$} & \multicolumn{4}{|c|}{ Rank 3} \\
\hline & $(\widetilde{y, \phi})$ & $\left(\widetilde{y, p_{\mathrm{t}}}\right)$ & $\left(\widetilde{\phi, p_{\mathrm{t}}}\right)$ & $\left(\widetilde{\left.y, \phi, p_{1}\right)}\right.$ \\
\hline 0 & $1.15 \pm 0.01$ & $1.125 \pm 0.011$ & $1.29 \pm 0.011$ & $1.13 \pm 0.01$ \\
\hline 1 & $1.22 \pm 0.01$ & $1.228 \pm 0.013$ & $1.352 \pm 0.015$ & $1.29 \pm 0.02$ \\
\hline 2 & $2.38 \pm 0.03$ & $1.256 \pm 0.016$ & $2.218 \pm 0.036$ & $2.76 \pm 0.06$ \\
\hline 3 & $4.97 \pm 0.11$ & $1.46 \pm 0.033$ & $3.13 \pm 0.08$ & $7.6 \pm 0.54$ \\
\hline 4 & $7.8 \pm 0.3$ & $1.79 \pm 0.10$ & $4.10 \pm 0.19$ & $17.1 \pm 4.1$ \\
\hline 5 & $9.8 \pm 1.0$ & $2.13 \pm 0.40$ & $4.75 \pm 0.63$ & \\
\hline 6 & $11.7 \pm 3.2$ & $2.3 \pm 1.4$ & $3.96 \pm 1.8$ & \\
\hline
\end{tabular}

sions. Three-dimensional factorial moments do not show saturation even though the numbers $M_{\bar{y}}$ of bins in which the $X$ interval has been divided are the same as for the one- and two-dimensional analyses. 


\subsection{EFFECTS OF RESONANCE DECAYS AND BOSE-EINSTEIN CORRELATIONS}

Resonance decays affect the factorial moments. Results from the CELLO collaboration have shown that this contribution is relevant in $\mathrm{e}^{+} \mathrm{e}^{-}$collisions [14] at lower energy.

The effect of resonance decays has been studied using the JETSET PS Monte Carlo with default settings, that describes the data reasonably well. Samples of 100000 hadronic events have been generated (i) switching off the $\pi^{0}$ decays, and (ii) switching off $\pi^{0}, \omega, \rho, \eta$ and $\eta^{\prime}$ decays.

Factorial moments are mostly affected by the $\pi^{0}$ Dalitz decay $\left(\pi^{0} \rightarrow \mathrm{e}^{+} \mathrm{e}^{-} \gamma\right)$ both in their absolute values and in their shape at high $M$. The other resonance decays mostly affect the absolute value, uniformly in $M$.

The most relevant effect in one-dimensional analyses is at low $p_{t}$, where the power-law behaviour at high $M$ shown in fig. 2 disappears when switching off the $\pi^{0}$ Dalitz decay.

The other one-dimensional analyses are not essentially affected by resonance decays, second- and third-rank factorial moments being increased by 1-2\% and $2-5 \%$, respectively.

In two and three dimensions the $\pi^{0}$ decays dramatically affect the factorial moments of second rank, whereas the third rank is relatively unaffected. In fig. 9 are shown results on one-dimensional $\tilde{y}$, two- and three-dimensional factorial moments obtained with JETSET PS Monte Carlo with default setting, with and without the contribution of resonance decays.
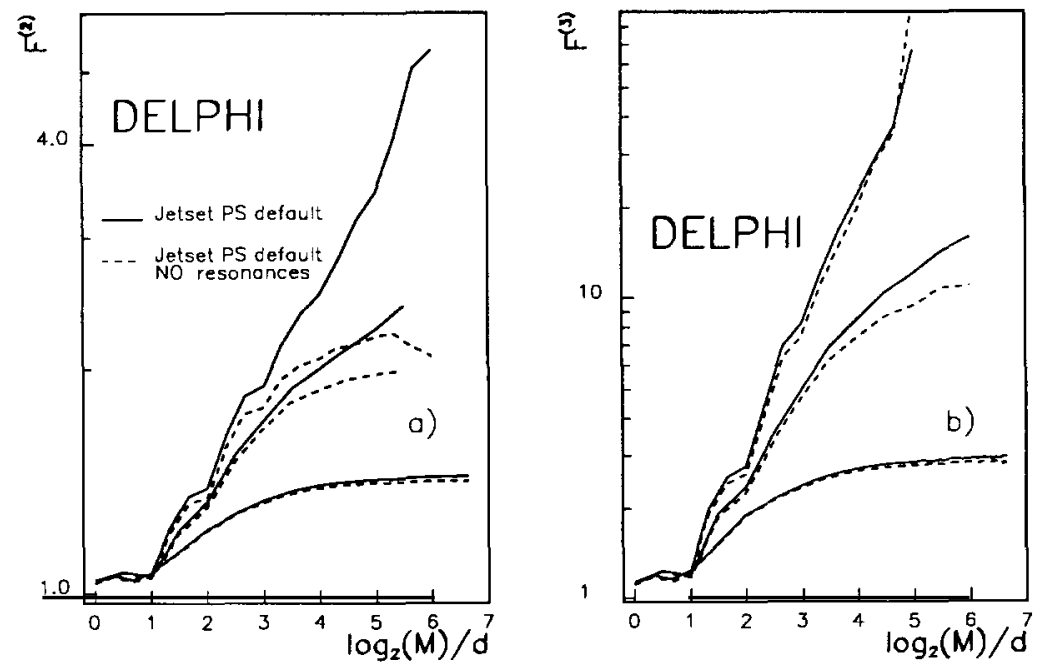

Fig. 9. The effect of resonance decays on factorial moment: a Monte Carlo study. Factorial moment for $\tilde{y},(\overline{y, \phi})$ and $\left(\widetilde{y, \phi, p_{\mathrm{t}}}\right)$ distributions as in fig. 8. JETSET 7.2 PS Monte Carlo prediction for default settings with (continuous line) and without (dashed lines) $\pi^{0}, \omega, \eta, \eta^{\prime}$ and $\rho$ decays. 
The influence of Bose-Einstein correlations on factorial moments has been investigated. The comparison of one-dimensional factorial moments for same sign particles with respect to random sign particles did not show differences in the first Delphi analysis [1]. However, this test is not conclusive since it ignores other correlations which can be different for the two sets of particles. Comparison with generators including a description of Bose-Einstein correlations has been done using JETSET PS Monte Carlo program. No improvement in the agreement of generators with data has been found. In fact, the Bose-Einstein correlations have no entirely satisfactory description in the generators [33,34]. So no final conclusion can be drawn on the eventual effect of Bose-Einstein correlations on intermittency and this remains a subject of further study.

\section{Conclusions}

An analysis of factorial moments of distributions of $Z^{0}$ hadronic final states, based on a statistics 10 times larger than previously published [1], confirms that Parton Shower models give a reasonable overall description of the data.

Analyses of events in different jet topologies and different intervals of $p_{t}$ show that fluctuations in the one-dimensional study come mainly from hard QCD effects.

The presence of intermittency, as defined in this paper, is not evident in one-dimensional projections, but becomes more evident in the two and three-dimensional analyses.

Influences of resonance decays have been studied by Monte Carlo simulation. Factorial moments in the $\tilde{y}$-variable are affected at low $p_{\mathrm{t}}$ as are the second-rank factorial moments in the two- and three-dimensional analyses.

No evidence for new physics is found because the models adequately describe the data.

We are greatly indebted to our technical collaborators and to the funding agencies for their support in building and operating the DELPHI detector, and to the members of the CERN-SL Division for the excellent performance of the LEP collider.

Discussions with A. Bialas, N. Geddes, A. Giovannini, R. Hwa, W. Kittel, N. Lieske, B. Buschbeck, W. Ochs, R. Peschanski and J. Seixas have helped considerably in clarifying the interpretation of the results.

\section{References}

[1] DELPHI Collaboration, P. Abreu et al., Phys. Lett. B247 (1990) 137;

A. De Angelis, Mod. Phys. Lett. A5 (1990) 2395 
[2] A. De Angelis and N. Demaria, in Proc. Ringberg Workshop, ref. [13]

[3] A. Bialas and R. Peschanski, Nucl. Phys. B273 (1986) 703; B308 (1988) 857

[4] G. Paladin and A. Vulpiani, Phys. Rep. 56 (1987) 147

[5] TASSO Collaboration, W. Braunschweig et al., Phys. Lett. B231 (1989) 548

[6] T. Sjöstrand, Comput. Phys. Commun. 27 (1982) 243; 28 (1983) 229;

T. Sjöstrand and M. Bengtsson, Comput. Phys. Commun. 43 (1987) 367

[7] G. Marchesini and B.R. Webber, Nucl. Phys. B238 (1984) 1

[8] P. Hoyer et al., Nucl. Phys. B161 (1979) 349

[9] B. Buschbeck, P. Lipa and R. Peschanski, Phys. Lett. B215 (1988) 788 [Based on data of the HRS collaboration, assuming that multiplicity follows the negative binomial distribution]

[10] HRS Collaboration, S. Abachi et al., Study of intermittency in $\mathrm{e}^{+} \mathrm{e}^{-}$annihilations at $29 \mathrm{GeV}$, contributed to XXV Conf. on High energy physics, Singapore, August 1990, ed. K.K. Phua and Y. Yamaguchi (World Scientific, Singapore)

[11] B. Bambah et al., QCD generators for LEP, Proc. 1989 Workshop on $Z^{0}$ Physics at LEP 1, CERN 89-08, Vol. 3, p. 143, ed. G. Altarelli, R. Kleiss and C. Verzegnassi

[12] DELPHI Collaboration, P. Aarnio et al., Nucl. Instrum Methods A303 (1991) 233

[13] R.C. Hwa, W. Ochs and N. Schmitz, ed., Proc. Ringberg Workshop on Multiparticle production fluctuations and fractal structure, Rindberg, Germany, June 1991 (World Scientific, Singapore, to be published)

[14] CELLO Collaboration, H.J. Behrend et al., Phys. Lett. B256 (1991) 97; O. Podobrin, DESY 91-028 (April 1991); in Proc. Ringberg Workshop, ref. [13]

[15] OPAL Collaboration, M.Z. Akrawy et al., Phys. Lett. B262 (1991) 351; N. Geddes, in Proc. Ringberg Workshop, ref. [13]

[16] ALEPH Collaboration, D. Decamp et al., Z. Phys. C53 (1992) 21;

N. Lieske, in Proc. Ringberg Workshop, ref. [13];

V. Raab, in Proc. Ringberg Workshop, ref. [13]

[17] N. Schmitz, Proc. XXI Int. Symp. on Multiparticle Dynamics, Wuhan, China, September 1990 (World Scientific, Singapore, to be published);

A. De Angelis, P. Lipa and W. Ochs, UDPHIR 91/06/AA (October 1991), to be published in Proc. LP-EPS Conf., Geneva 1991;

A. Bialas, CERN-TH.5791/90 (July 1990);

R. Peschanski, CERN-TH.5891/90 (October 1990)

[18] A. Bialas and R. Peschanski, Nucl. Phys. B308 (1988) 857

[19] W. Ochs and J. Wosiek, Phys. Lett. B214 (1988) 617;

W. Ochs and J. Wosiek, Phys. Lett. B232 (1989) 271

[20] P. Carruthers and I. Sarcevic, Phys. Rev. Lett. 63 (1989) 1562

[21] DELSIM User Manual, DELPHI 87-96 PROG-99, Geneva (July 1989); DELSIM Reference Manual, DELPHI 87-98 PROG-100, Geneva (July 1989)

[22] DELPHI Collaboration, P. Aarnio et al., Phys. Lett. B240 (1990) 271

[23] OPAL Collaboration, M.Z. Akrawy et al., Z. Phys. C47 (1990) 505

[24] ALEPH Collaboration, D. Decamp et al., Phys. Lett. B234 (1990) 209

[25] DELPHI Collaboration, P. Abreu et al., Z. Phys. C50 (1991) 185; C52 (1991) 271; CERN-PPE/92064 (April 1992)

[26] DELPHI Collaboration, Comparison of data with QCD models, contributed to LP-HEP Conf., Geneva, July 1991 ;

W. de Boer, H. Fürstenau and J.H. Köhne, Z. Phys. C49 (1991) 141

[27] L. Lönnblad, LU TP 89-10, Lund (1989)

[28] G. Ekspong, discussion remark (unpublished), Workshop on Intermittency in multiparticle collisions, Santa Fé, NM, March 1990

[29] A. Bialas and M. Gazdzicki, Phys. Lett. B252 (1990) 483; W. Ochs, Z. Phys. C50 (1991) 339

[30] EHS /NA22 Collaboration, N.M. Agababyan et al., Phys. Lett. B261 (1991) 165

[31] G. Marchesini and B.R. Webber, Nucl. Phys. B310 (1988) 461;

G. Marchesini et al., Cavendish-HEP-90/26 
[32] JADE Collaboration, W. Bartel et al., Z. Phys. C33 (1986) 23;

S. Bethke et al., Phys. Lett. B213 (1988) 235

[33] B. De Lotto, Proc. XXth Int. Symp. on Multiparticle Dynamics, Dortmund, September 1990, ed. R. Baier and D. Wegener (World Scientific, Singapore) p. 514; DELPHI Collaboration, P. Abreu et al., CERN-PPE/92-75 (May 1992)

[34] OPAL Collaboration, P. Acton et al., Phys. Lett. B267 (1991) 154

[35] W. Ochs, Phys. Lett. B247 (1990) 101;

A. Bialas and J. Seixas, Phys. Lett. B250 (1990) 161

[36] L3 Collaboration, B. Adeva et al., Z. Phys. C55 (1992) 39 